\title{
CONTEXT CLASSIFICATION AND ADAPTIVE PREDICTION FOR LOSSLESS IMAGE CODING
}

\author{
Farshid Golchin ${ }^{*}$ and Kuldip K. Paliwal \\ School of Microelectronic Engineering \\ Griffith University \\ Brisbane, QLD 4111, Australia \\ F.Golchin, K.Paliwal@me.gu.edu.au
}

\begin{abstract}
In this paper, we combine a context classification scheme with adaptive prediction and entropy coding to produce an adaptive lossless image coder. In this coder, we maximize the benefits of adaptivity using both adaptive prediction and entropy coding. The adaptive prediction is closely tied with the classification of contexts within the image. These contexts are defined with respect to the local edge, texture or gradient characteristics as well as local activity within small blocks of the image. For each context an optimal predictor is found which is used for the prediction of all pixels belonging to that particular context. Once the predicted values have been removed from the original image, a clustering algorithm is used to design a separate, optimal entropy coding scheme for encoding the prediction residual. Blocks of residual pixels are classified into a finite number of classes and members of each class are encoded using the entropy coder designed for that particular class. The combination of these two powerful techniques produces some of the best lossless coding results reported so far.
\end{abstract}

\section{INTRODUCTION}

Image coding is an essential component of many digital transmission and storage systems. Generally speaking, image coding algorithms can be divided into two categories: lossy and lossless. Lossy compression techniques which are by far the more popular, result in coding distortions (or artifacts) which are not tolerable in certain applications. In areas such as medical image coding or some satellite imaging, coding artifacts can have potentially serious consequences or result in loss of data obtained at a great cost.

It is in these areas that lossless image coding methods are utilized. Although lossless methods offer significantly lower compression ratios, the fact that they preserve the original data, has made them indisposable. In recent years, a great deal of the research in image coding has concentrated on lossy methods. The development of better transforms, quantizers and particularly adaptivity in coders has resulted in significant advances in this area.

An adaptive coder is able to adapt its workings to the local characteristics within the different regions of an image. Adaptive lossless image coding is by no means a new

\footnotetext{
*The first author is supported in part by a CSIRO Division of Telecomm. and Industrial Physics postgraduate scholarship.
}

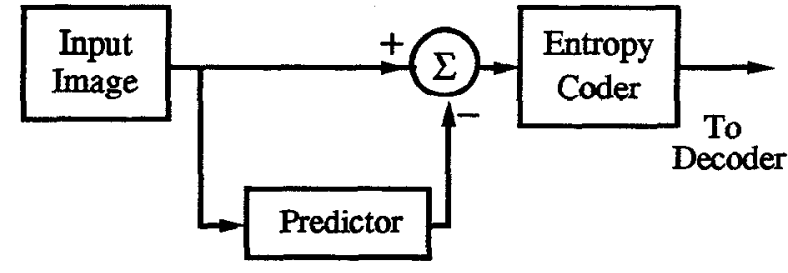

Figure 1. A DPCM-based lossless coder

area. The use of "contexts" [1] in various forms has demonstrated significant improvements in lossless compression results. However, many of these techniques are based on heuristics and often do not exploit the full benefits offered by adaptivity.

A lossless image coder in its simplest form, is implemented using a fixed predictor and a fixed entropy coder as shown in Fig. 1. The predictor, makes a prediction for each pixel based on previously transmitted values. The predicted values are then subtracted from the original values, thus leaving the prediction error (or residual). An entropy coder is then used to encode the residual signal.

Adaptivity can be provided by making either or both of these components adaptive. An adaptive predictor is able to adapt to the characteristics of various textures or the direction of edges and hence produce more accurate predictions. An adaptive entropy coder, on the other hand, can adjust its workings to better match the local statistics of an image and produce shorter-length codes.

\section{CONTEXT CLASSIFICATION AND ADAPTIVE PREDICTION}

In this paper, we concentrate on one particular type of adaptive prediction which is performed by switching among a finite number of predictors. The decision of which predictor to use, is made for each pixel being encoded and is solely based on previously transmitted pixel values.

The difficulty with this type of adaptive prediction schemes is in the trade-off that exists between the number of predictors and computational complexity. Since the appropriate predictor is often selected using an exhaustive search [2] the computational complexity is high and can only be reduced by limiting the number of predictors. We have previously presented an optimal scheme for the design of a small number of predictors [2]; however, this scheme requires a computationally intensive design process. 
The main performance obstacle in the path of the adaptive prediction schemes mentioned above is the exhaustive search required to map the local context from pixel domain to an appropriate predictor. The performance can be significantly improved if a faster mapping is used. Such a mapping has been recently proposed by $\mathrm{Wu}$ [5]. The coder named CALIC [5], [6], has many distinct features which contribute to its state-of-the-art performance; however its most significant (and possibly understated) feature is its Context Classification (or Quantization).

In CALIC a fast method is used to classify the causal neighborhood (context) of the next pixel to be encoded into a finite set of contexts. For each of the possible contexts, a bias value (a scalar) is kept which is added to the prediction made by a less sophisticated linear predictor in order to improve its performance. CALIC assumes that the first predictor is consistently repeating the same prediction error over the same context which can be compensated for using the bias value.

In this paper, we take a slightly different approach and set out to exploit this fast mapping to the fullest. We do so by using context classification to select a linear predictor from a set of 1024 linear predictors. The classification is performed on the context $C=(w, w w, n w, n, n n, n e, 2 n-$ $n n, 2 w-w w$ ) [6], where each of the elements is the pixel in that direction (north, south, ...) with respect to the pixel being encoded, as shown in Fig. 2.

If each element in $\mathrm{C}$ is larger than or equal to the mean of $C$, then a corresponding bit is set to 0 , otherwise the bit is set to 1 . This produces the 8-bit binary string $S=\left(s_{1}, s_{2}, \ldots, s_{8}\right)$ which describes the shape of the context of the pixel being encoded.

A measure of local energy is also quantized into one of 4 levels using Lloyd-Max [8] type scalar quantizer. This type of classification is quite similar to what is done in GainShape vector quantization. In this case the binary string $S$ describes the "shape" of the region while the energy level describes the "gain" of the particular region. Combining the "shapes" and "gains" together provides a mapping from pixel domain into 1024 different contexts.

We design an optimum linear predictor for each of the 1024 contexts using either training data or the image to be encoded. In coding, the context of each pixel is classified and then the appropriate predictor is used for the prediction of that particular pixel. It should be noted that the predictors for each context are designed using as MeanSquared-Error (MSE) criterion.

Although it would be more intuitive to design the predictors according to a minimum entropy criterion, the coding advantage (particularly in the presence of an adaptive entropy coding system) of such predictors over simple MSE optimized predictors is negligible. Hence, considering the additional computational complexity in designing minimum-entropy predictors, MSE optimized predictors were selected. The coefficients of these predictors can be simply calculated by solving the well-known Wiener-Hopf equations.

In the following section, we briefly describe the adaptive entropy coding method used in our lossless coder.

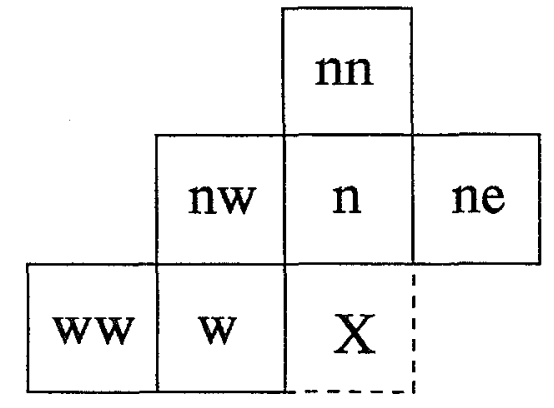

Figure 2. The Prediction context of pixel $X$

\section{THE MINIMUM-ENTROPY CLUSTERING ALGORITHM}

The adaptive prediction scheme described in the previous section, decorrelates neighboring pixels in the image. However, upon inspecting the residual image, it becomes clear that large values of residuals (prediction error) tend to be confined to certain areas while small residual values are also grouped together.

This non-uniformity in the local statistics of the areas within the image can be exploited in entropy coding. This is done through using different entropy coders, each of which is matched to a particular type of region in the residual image. The question remains as to how the different areas within the image can be classified into different types and how the entropy coders can be designed. To this end, we utilize the Minimum-Entropy Clustering (MEC) algorithm.

Our aim in the use of the Minimum-Entropy Clustering algorithm is to classify blocks of samples and then encode the samples in each block using a suitable entropy coder. The classification and design of the entropy coders should be performed in a way such that the overall entropy is minimized.

In order to design a coding system with $N$ entropy coders, the MEC algorithm operates as follows:

1. Initialization: $N$ probability distribution functions (PDF's) are defined. These PDF's will define the initial classes.

2. Minimum-Code-Length classification: Each block of samples $B=\left(b_{0}, b_{1}, \ldots, b_{m-1}\right)$ is classified as belonging to class $C$ such that the code length (after entropy coding) $L=-\sum_{i=0}^{m-1} \log _{2} p\left(b_{i} \mid C\right)$ is minimized. This is similar to a nearest neighbor selection in VI design.

3. Re-Estimate class statistics: Estimate the new class PDF's. This will ensure that the class statistics are matched to those of the samples in that class and hence the entropy is further reduced. This step is similar to a centroid calculation in $V Q$ design.

4. Iteration: Stop if a maximum number of iterations is reached or the classes have converged. Otherwise, go to step 2 .

Steps 2 and 3 form the core of the MEC algorithm. In each of these two steps, the overall entropy is reduced. Although there is no guarantee of convergence to a global minimum, it was found that the algorithm quickly converges 
to a satisfactory minimum. The speed of the convergence depends greatly on the initialization procedure used. However, in almost all cases, it was found that the algorithm converges to the same minimum regardless of the initial settings.

There are a number of choices available for the initial classification. However, a better definition of the initial classes can significantly reduce the number of iterations required. Since we wish to group together blocks with similar statistics, a reasonable choice for initial classification would be classification based on the variance of the blocks. To do so, we choose a classification similar to that used by Chen and Smith [3]. The variance of each block is estimated and the blocks are listed in the order of increasing (or decreasing) variance.

Using the sorted list, $m-1$ threshold values (of variance) are selected and used to classify the blocks into $m$ classes. After this classification, the class PDF's are estimated and used to define the initial classes.

This algorithm may be used in either a parametric or a non-parametric form. In its parametric form, all distributions are modeled as Generalized Gaussian (GG) distributions [4] whose shape parameter and variance are estimated. In this case, step 3 of the algorithm becomes a maximumlikelihood estimation of the shape parameter and variance. The main disadvantage of using the parametric form of the MEC algorithm is the additional processing required in the calculation of probabilities (Step 2) and the parameter estimation (Step 3).

In the non-parametric version of the MEC algorithm, frequency tables are maintained for each of the classes. These frequency tables are updated in step 3 of the algorithm. After the MEC algorithm has converged, these frequency tables are used to design the entropy coders. It is for this reason that the frequency tables must also be known at the decoder. In the parametric case, the probabilities can be efficiently described to the decoder by transmitting two parameters (shape and variance) per class.

In the non-parametric version, the frequency tables must be explicitly transmitted. To reduce the amount of information, we may take advantage of the symmetry in the frequency tables. One half of each frequency table is quantized using 6-8 bits and then transmitted to the decoder. If adaptive entropy coders are used, the probability tables are quickly adapted to match the source statistics and any mismatches caused by quantization rapidly disappear.

There is a trade-off between the parametric and the non-parametric versions of the MEC algorithm. The nonparametric version offers faster execution times at the expense of added side-information (frequency tables). However, it should be noted that in terms of compression the two versions of the MEC algorithm produce quite similar results. The results in this paper have been obtained using the non-parametric version of the MEC algorithm.

\section{A CONTEXT CLASSIFICATION BASED IMAGE CODER}

In this section, we examine the design of the adaptive predictor and the entropy coders used to encode the prediction residuals. As mentioned in the previous section, the predic- tors can be designed either for a training set of images or specifically for the image to be encoded. There is a trade-off between the the two methods which parallels the trade-offs experienced in quantizer design.

If a training set of images are used, the training is performed offline, however the predictors are not designed for the particular image and the predictors are slightly inferior in performance. Alternatively, if the predictors are designed for the image to be encoded, then some online training is required and the predictor coefficients must be transmitted to the receiver.

In this paper, we examine both of the above-mentioned scenarios and compare their performance. The training algorithm for the predictors is as follows:

1 For each pixel in the image (Training image, or image to be encoded) the context $\mathrm{C}$ is found.

2 We calculate and remove the average value of $\mathrm{C}$ from the pixels in $\mathrm{C}$.

3 Take the sign of each of the mean removed values as $+=0$ and $-=1$ to make the 8 -bit binary shape vector.

4 Calculate the standard deviation of the values in C.

5 Quantize the Standard deviation into one of 4 levels.

6 The pixel is classified as belonging to 1024 contexts depending on its shape and gain.

7 Update autocorrelation values for that context.

The standard deviation values are quantized using a Lloyd-Max quantizer [8]. Once the classification has been completed and the autocorrelation values are finalized, the autocorrelation values are used to design a 4th-order optimum linear predictor for each context. If a particular context appears rarely or not at all (in the training set), then it is allocated a trivial second order predictor with two coefficients of 0.5 .

It should also be noted that if the predictors are specifically designed for the image to be encoded, their coeffcients must be transmitted as side information and contribute around 0.01 to $0.02 \mathrm{bpp}$ to the overall bit-rate. All predictor coefficients are quantized using a second LloydMax quantizer and at a resolution of 10 bits per predictor coefficient.

It is interesting to note that within a typical image, only $100-300$ of the possible 1024 contexts appear. This is a significant advantage with respect to the transmission of the predictor coefficients, since only a small portion of the predictor coefficients need to be transmitted.

The MEC algorithm was used to define the classification scheme and design the entropy coders. Classification was made on blocks of $8 \times 8$ pixels since it was experimentally found to produce the best results. The $8 \times 8$ blocks were classified into 16 classes and hence encoded using 16 different entropy coders. As mentioned previously, along with the classification scheme, the frequency tables for the entropy coders must also be transmitted to the decoder. For a $512 \times 512$ pixel image this results in an added bit-rate of 


\begin{tabular}{|c|c|c|c|}
\hline \multirow{2}{*}{$\begin{array}{c}\text { Coded } \\
\text { Image }\end{array}$} & \multicolumn{3}{|c|}{ Coding Method } \\
\cline { 2 - 4 } barb & CCBAC-TS & CCBAC-SI & CALIC [6] \\
\hline fruit & 4.34 & 4.29 & 4.43 \\
\hline lena & 3.94 & 4.36 & 4.55 \\
\hline man & 4.38 & 3.90 & 4.05 \\
\hline goldhill & 4.70 & 4.35 & 4.43 \\
\hline
\end{tabular}

Table 1. A comparison of lossless compression results (quoted in bpp)

0.01 bpp. For a $256 \times 256$ pixel image this overhead increases to approximately $0.04 \mathrm{bpp}$.

\section{RESULTS AND CONCLUSIONS}

The coding results are listed in Table 1 . The Context Classification Based Adaptive Coder trained on a Training Set is referred to as CCBAC-TS and the coder trained on the Specific Image is referred to as CCBAC-SI. A set of ten images, excluding the training set was used for training the CCBAC-TS. The values quoted in the table are based on actual file sizes and include all overheads.

The results obtained from CALIC [6], were also provided for comparison. From Table 1, it is clear that both proposed methods significantly outperform CALIC which is considered to be the state-of-the-art in lossless image coding.

As expected, the image specific training algorithm CCBAC-SI outperforms the pre-trained predictors used in CCBAC-TS. What is surprising however, is the small difference between the two sets of results. This suggests that in many cases, the pre-trained predictors may be advantageous, since they require no "online" training.

The use of "off-line" training (using a training set of images), is even more appropriate when the coder is being used on satellite or medical images. For instance, if the coder is going to be used in encoding $\mathrm{X}$-ray images, then the predictors can be trained using a suite of X-ray images.

Both of the tested coders outperform CALIC. We must, however, acknowledge the increased computational complexity of both coders compared to CALIC. Work is currently in progress toward further optimizing the speed of the presented algorithms. Also, future research will be performed into the context activity (standard deviation) quantizer which is a Lloyd-Max quantizer at this time. Using a more suitable quantizer will almost certainly improve coding results.

The prediction scheme presented in this paper, demonstrates the advantages of using multiple predictors and the fast context classification scheme presented. The fact that the context classification scheme does not use an exhaustive search allows a large number of contexts to be utilized. The combination of this type of prediction with an adaptive entropy coding scheme such as the MEC algorithm was shown to produce some of the best results obtained in image coding to date.

\section{REFERENCES}

[1] T.V. Ramabadran and K. Chen, "The use of contextual information in the reversible compression of medi- cal images," IEEE Trans. Medical Imaging, vol. 11, pp. 185-195, June 1992.

[2] F. Golchin and K.K. Paliwal, "Clustering-based adaptive prediction and entropy coding for use in lossless image coding," Accepted for ICIP-97, October 1997.

[3] W.H. Chen and C.H. Smith, "Adaptive coding of monochrome and color images," IEEE Trans. on Commun., vol. COM-25, pp. 1285-1292, November 1977.

[4] N. Farvardin and J.W. Modestino, "Optimum quantizer performance for a class of non-Gaussian memoryless sources," IEEE Trans. Inform. Theory, vol. IT-30, pp. 485-497, May 1984.

[5] X. Wu, "Context selection and quantization for lossless image coding," Proc. Data Compression Conf. '95, March 1995.

[6] X. Wu and N. Memmon, "CALIC - A context based lossless image codec," Proc. Int. Conf. on Acoust., Speech, Signal Processing, Atlanta, GA, pp. 1891-1894, May 1996.

[7] Y. Linde, A. Buzo and R.M. Gray, "An algorithm for vector quantizer design," IEEE Trans. Comm., COM28, pp. 84-95, January 1980.

[8] S.P. Lloyd, "Least squares quantization in PCM," IEEE Trans. Inform. Theory, IT-28, pp. 127-135, March 1982.

[9] F. Golchin and K.K. Paliwal, "Minimum-entropy clustering and its application to lossless image coding," Accepted for ICIP-97, October 1997. 